\title{
The IRE1a-XBP1s pathway promotes insulin-stimulated glucose uptake in adipocytes by increasing PPARY activity
}

\author{
Yoon Mi Cho ${ }^{1}$, Dong Hee Kim', Kyung Hye Lee ${ }^{1}$, Seong-Whan Jeong ${ }^{1}$ and Oh-Joo Kwon ${ }^{1}$
}

\begin{abstract}
The peroxisome proliferator-activated receptor- $\gamma$ (PPARY) improves whole-body insulin sensitivity by regulating the adipogenic and metabolic functions of mature adipocytes. We have previously demonstrated that an active splice variant of X-box binding protein 1 (XBP1s) enhances PPARy expression during adipogenesis. In this study, we investigated the role of XBP1s, particularly with respect to PPARY, in the mechanisms underlying insulin sensitivity in mature adipocytes. Insulin was able to stimulate XBP1s generation by activating inositol-requiring enzyme 1 (IRE1) $a$ and was also able to increase its transcriptional activity by inducing nuclear translocation. XBP1s also upregulated the levels of phosphorylated IRS1 and AKT, demonstrating a positive feedback regulatory mechanism linking insulin and XBP1s. XBP1s enhanced the expression of fibroblast growth factor 21 and, in turn, increased PPARY activity, translocation of GLUT4 to the cell surface, and glucose uptake rate in adipocytes. In addition, XBP1s abolished palmitate-induced insulin resistance in adipocytes by increasing adiponectin secretion, repressing the secretion of proinflammatory adipokines such as leptin, monocyte chemoattractant protein 1, and tumor necrosis factor a, and decreasing fatty acid release. These findings provide a novel mechanism by which XBP1s stimulate insulin sensitivity in adipocytes through fibroblast growth factor 21 induction and PPARY activation.
\end{abstract}

\section{Introduction}

Adipose tissue is a key energy storage organ and is also becoming known as an endocrine organ with multiple roles in metabolism and the regulation of whole-body energy homeostasis ${ }^{1}$. In conditions characterized by excessive energy, such as obesity, insulin resistance is locally initiated in adipose tissue and subsequently progresses to systemic insulin resistance. This systemic resistance is thought to be a major risk factor for metabolic disorders such as type 2 diabetes, fatty liver disease, atherosclerosis, and some cancers $^{2}$. Consequently, understanding how insulin sensitivity is regulated in adipocytes may be of therapeutic value for the treatment of obesity-related metabolic diseases.

Peroxisome proliferator-activated receptor- $\gamma$ (PPAR $\gamma)$ is a ligand-activated transcription factor that belongs to the

\footnotetext{
Correspondence: Oh-Joo Kwon (ojkwon@catholic.ac.kr)

${ }^{1}$ Department of Biochemistry, College of Medicine, The Catholic University of Korea, Seoul, Republic of Korea
}

nuclear receptor superfamily. PPAR $\gamma$ is most abundantly expressed in adipose tissue, and both its expression and activity are regulated by insulin ${ }^{3}$, suggesting that PPAR $\gamma$ functions within adipose tissue to regulate insulin sensitivity. PPARY activation exerts systemic insulinsensitizing effects by inducing the expression of several genes involved in the insulin signaling cascade as well as the synthesis of beneficial adipokines within adipose tissue $^{4}$. In addition, PPAR $\gamma$ is widely accepted as a master regulator of adipocyte differentiation (adipogenesis), an important mechanism for maintaining insulin sensitivity in adipose tissue and preventing ectopic fat accumulation and cardiovascular risk $^{5}$. Therefore, synthetic PPAR $\gamma$ ligands are widely used in the treatment of insulin resistance-related pathological conditions ${ }^{6}$; however, the detailed mechanisms underlying PPAR $\gamma$ activation in adipose tissue are poorly understood.

$\mathrm{X}$-box binding protein 1 (XBP1) is a member of the bZIP family of transcription factors. XBP1 is converted to 
its transcriptionally active isoform (XBP1s) by inositolrequiring enzyme 1 (IRE1) $\alpha$-mediated unconventional splicing of XBP1 $\mathrm{mRNA}^{7}$. Most of the knowledge regarding the role of XBP1s in insulin sensitivity comes from in vitro studies of adipogenesis. In this context, we have also previously identified a set of pro-adipogenic mechanisms by which XBP1s stimulates PPAR $\gamma 2$ expression directly and indirectly by regulating Wnt10b and miR-148a, respectively ${ }^{8-10}$. Findings from a relatively thorough in vitro investigation suggest that XBP1s plays a pro-adipogenic role. However, its role in mature adipocytes, which make up almost $30 \%$ of the cellular content of adipose tissue, is widely unknown. In relation to insulin signaling, a number of in vivo studies suggest that XBP1s is implicated in the regulation of whole-body insulin sensitivity. For example, both the nuclear translocation and activity of XBP1s are reduced in the livers of obese mice ${ }^{11}$; this decrease in XBP1s activity is associated with the development of insulin resistance and type 2 diabetes in obese mice ${ }^{12}$, while enhancing XBP1s activity increases hepatic insulin sensitivity and improves glucose homeostasis $^{12,13}$. However, the adipose-specific roles of XBP1s have not been fully addressed. To the best of our knowledge, only two studies have demonstrated adiposespecific in vivo roles of XBP1s, and these findings have not been consistent with each other. One study reported that adipocyte-specific deletion of XBP1s did not affect inflammation or metabolic homeostasis ${ }^{14}$. In contrast, a second study reported that XBP1s improves systemic glucose homeostasis by increasing adiponectin multimerization in an adipocyte-specific XBP1s gain-offunction mouse model ${ }^{15}$. Further studies are needed to elucidate the adipocyte-specific role of XBP1s in the regulation of insulin sensitivity.

In this study, we explored the insulin-sensitizing role of $\mathrm{XBP} 1 \mathrm{~s}$ and the underlying molecular mechanisms of this sensitization in differentiated mouse 3T3-L1 adipocytes. This study provides new insights into the role of XBP1s in the pathogenesis and treatment of metabolic disorders related to insulin resistance.

\section{Materials and methods}

\section{Culture and differentiation of 3T3-L1 cells}

Murine 3T3-L1 fibroblasts (American Type Culture Collection, Manassas, VA, USA) were maintained and differentiated into adipocytes as previously described ${ }^{9}$. Briefly, cells were maintained for 2 days in $4.5 \mathrm{gl}^{-1}$ glucose-containing Dulbecco's modified Eagle's medium (DMEM) supplemented with $10 \%$ normal calf serum (Gibco, Grand Island, NY, USA). Two days after reaching confluence (day 0 ), adipogenic differentiation was induced for 2 days in medium containing insulin $\left(1 \mu \mathrm{g} \mathrm{ml}{ }^{-1}\right.$; Sigma, St Louis, MO, USA), IBMX (0.5 mM; Sigma), and dexamethasone $(1 \mu \mathrm{M}$; Sigma). Then, the cells were cultured for 2 days in DMEM supplemented with $10 \%$ fetal bovine serum (FBS) (Gibco) and insulin $\left(1 \mu \mathrm{g} \mathrm{ml}^{-1}\right)$. Following this, the medium was changed to the DMEM/ 10\% FBS until day 8 .

\section{Genetic manipulation of XBP1s and FGF21 in adipocytes}

Differentiated 3T3-L1 adipocytes were transiently transfected by electroporation as follows. Cells were detached from the plates with trypsin/EDTA and resuspended in serum-free Opti-MEM (Gibco) prior to electroporation. Approximately 2.5 million cells were mixed with $20 \mathrm{nM}$ XBP1 (sense: GACCAUCACAUU GCCUAGA, antisense: UCUAGGCAAUGUGAUGGUC) or fibroblast growth factor 21 (FGF21; sense: AGAUG ACGACCAAGACACU, antisense: AGUGUCUUGGUC GUCAUCU) siRNA duplexes (Bioneer, Daejeon, Korea) or $1 \mu \mathrm{g}$ of a mouse XBP1s-overexpressing recombinant plasmid (pcDNA3.1-mXBP1s) ${ }^{9}$. Then, the cells were pulsed at $1300 \mathrm{~V}$ for $20 \mathrm{~ms}$ using the Neon ${ }^{\text {Tx }}$ Transfection System (Invitrogen, Carlsbad, CA, USA) and were immediately incubated with fresh media before reseeding. Experiments were conducted $48 \mathrm{~h}$ after electroporation.

\section{Measurement of glucose uptake rate}

3T3-L1 adipocytes were cultured overnight in serumfree DMEM in 12-well plates and then washed in phosphate-buffered saline (PBS) and incubated in glucose-free DMEM for $1 \mathrm{~h}$. Subsequently, $18.5 \mathrm{kBq}$ of 2deoxy-D- ${ }^{3} \mathrm{H}$-glucose (PerkinElmer, San Jose, CA, USA) and $0.1 \mathrm{mM} 2$-deoxyglucose were added. After $30 \mathrm{~min}$, the reaction was terminated with cytochalasin $\mathrm{B}(20 \mu \mathrm{M})$, and the cells were washed with ice-cold PBS. Cells were lysed for $10 \mathrm{~min}$ in $0.1 \%$ sodium dodecyl sulfate (SDS), and then aliquots of lysates were used for liquid scintillation counting and determination of protein concentration by BCA protein assay (Thermo Scientific, Rockford, IL, USA). Radioactivity was measured using a $\beta$-counter (PerkinElmer). Radioactivity was normalized to protein concentration and is presented as a percentage of basal level.

\section{Immunofluorescence microscopic examination}

3T3-L1 adipocytes were grown and differentiated on coverslips and then fixed with $4 \%$ paraformaldehyde for $20 \mathrm{~min}$. After washing with PBS for $10 \mathrm{~min}$, cells were blocked with $2 \%$ bovine serum albumin (BSA) in PBS for $30 \mathrm{~min}$. After washing again, cells were incubated with rabbit anti-mouse GLUT4 antibody (Merck Millipore, Darmstadt, Germany, \#07-1404) (1:100 dilution in 1\% BSA/PBS) overnight at $4{ }^{\circ} \mathrm{C}$. Cells were then washed and incubated for $1 \mathrm{~h}$ at room temperature with FITCconjugated anti-rabbit IgG (1:150 in $1 \%$ BSA/PBS). Nuclei were stained with DAPI (Invitrogen), and the images were visualized with an LSM 510 Meta confocal 
Table 1 Primer sequences for PCR analysis

\begin{tabular}{|c|c|c|c|c|}
\hline \multirow[t]{2}{*}{ Name } & \multicolumn{2}{|l|}{ Primer sequences $\left(5^{\prime}-3^{\prime}\right)$} & \multirow{2}{*}{$\begin{array}{l}\text { Size } \\
\text { (bp) }\end{array}$} & \multirow[t]{2}{*}{ Gene ID } \\
\hline & Sense & Antisense & & \\
\hline Adiponectin & TGTGTATCGCTCAGCGTTCAGTGT & AGAGAACGGCCTTGTCCTTCTTGA & 224 & NM_009605 \\
\hline aP2 & GAT GCC TाT GTG GGA ACC T & CTG TCG TCT GCG GTG ATT T & 230 & NM_024406 \\
\hline CD36 & CAG GTC TAT CTA CGC TGT GTT C & CAG GCT TTC CTT CTT TGC ATT T & 200 & NM_007643 \\
\hline ERdj4 & AGT CTG CCT CAG AGC GAC AAA TCA & GCC TCT TTG TCC TाT GCC ATT GGT & 203 & NM_013760 \\
\hline FGF21 & CTACACAGATGACGACCAAGA & ATCAAAGTGAGGCGATCCATAG & 205 & NM_020013 \\
\hline GLUT4 & TCG TGG CCA TAT TTG GCT TTG TGG & TAA GGA CCC ATA GCA TCC GCA ACA & 190 & NM_009204 \\
\hline Leptin & GGC TIT GGT CCT ATC TGT CTT AT & ATT CTC CAG GTC ATT GGC TAT C & 278 & NM_008493 \\
\hline LPL & ACGAGCGCTC CATTCATCTC TTCA & TCTTGCTGCT TCTCTTGGCT CTGA & 180 & NM_008509 \\
\hline MCP1 & GAA GGA ATG GGT CCA GAC ATA C & TCA CAC TGG TCA CTC CTA CA & 200 & NM_011333 \\
\hline $\mathrm{p} 58^{\mathrm{IPK}}$ & $\pi \Pi$ CAC GCT GCA GTT GAT GGT GAC & TCT TCT GCT TCG TCA AGC TTC CCT & 200 & NM_008929 \\
\hline PPARyt & CGA GCC CTG GCA AAG CAT TTG TAT & AAA TTC GGA TGG CCA CCT CTT TGC & 211 & NM_001308354 \\
\hline PPARY2 & CGCTGATGCACTGCCTATGA & AGAGGTCCACAGAGCTGATTCC & 101 & NM_011146 \\
\hline TNF & CGTCAGCCGATTTGCTATCT & AGCAATGACTCCAAAGTAGACC & 232 & NM_013693 \\
\hline $\mathrm{XBP} 1 \mathrm{t}$ & AGC AGC AAG TGG TGG ATT TGG AAG & AAG AGG CAA CAG TGT CAG AGT CCA & 299 & NM_013842 \\
\hline XBP1s & CTGAGGTCCGCAGCAGGT & TGTCAGAGTCCATGGGAAGA & 66 & NM_001271730 \\
\hline 36B4 & GCTCCAAGCAGATGCAGCA & CCGGATGTGAGGCAGCAG & 143 & NM_007475 \\
\hline $\begin{array}{l}\text { fgf21 promoter } \\
\text { (ChIP assay) }\end{array}$ & CTC AGA CCA AGG AGC ACA GA & TGA ACG CAG AAA TAC CAG AAT & 257 & ENSMUST00000033099 \\
\hline pEGFP-mXBP1 & $\begin{array}{l}\text { TTG AAT TCT GAT GGT GGT GGT GGC AGC } \\
\text { GGC GCC GA }\end{array}$ & $\begin{array}{l}\text { TTG TCG ACA GGC CTG CAC CTG CTG CAG } \\
\text { AGG TGC ACA }\end{array}$ & - & - \\
\hline $\begin{array}{l}\text { pcDNA-WT- } \\
\text { mXBP1u }\end{array}$ & TTG CGG CCG CAT GGT GGT GGT GGC A & $\begin{array}{l}\text { TTA AGC TTG ACA CTA ATC AGC TGG GGG AAA } \\
\text { AGT TC }\end{array}$ & - & - \\
\hline $\begin{array}{l}\text { pcDNA-Mut- } \\
\text { mXBP1u }\end{array}$ & $\begin{array}{l}\text { ACT CAG ACT ATG TGC ACC TाT GCG ACA GGT } \\
\text { GCA GGC CCA GT }\end{array}$ & $\begin{array}{l}\text { AAC TGG GCC TGC ACC TGT CGC AAA GGT } \\
\text { GCA CAT AGT CTG AGT }\end{array}$ & - & - \\
\hline
\end{tabular}

laser-scanning microscope (Zeiss, Oberkochen, Germany) at $\times 400$ magnification.

\section{Real-time PCR}

Total RNA was extracted using TRIzol reagent (Invitrogen), and complementary DNA was synthesized using the PrimeScript RT kit (TaKaRa, Shiga, Japan). Realtime PCR analysis was performed using an ABI 7300 thermal cycler (Applied Biosystems, Foster City, CA, USA) with SYBR Green Q-PCR Master Mix (TaKaRa). The $\Delta \Delta C t$ method was used to calculate the relative mRNA levels, using the $36 \mathrm{~b} 4$ gene as an internal control. The primer sequences used for PCR are listed in Table 1.

\section{In vitro insulin resistance model}

To establish an insulin-resistant state in vitro, cells were pretreated with low serum-containing media (0.25\% FBS) overnight, washed with PBS, and then incubated with palmitate (200 $\mu \mathrm{M}$, Sigma) for $24 \mathrm{~h}$. Palmitate-stimulated lipolysis was determined by measuring free glycerol content in the culture media using a serum triglyceride determination kit (Sigma) as described previously ${ }^{9}$. Briefly, the culture media was centrifuged at $16,000 \mathrm{~g}$ for $10 \mathrm{~min}$, and $10 \mu \mathrm{l}$ of glycerol standard or sample was mixed with $500 \mu$ l of kit reagents (Sigma). Glycerol concentration was measured at $540 \mathrm{~nm}$ and normalized to total cellular protein.

\section{Enzyme-linked immunosorbent assay}

Media was collected and centrifuged for $10 \mathrm{~min}$ at $800 \mathrm{~g}$. The supernatant fraction was stored at $-70{ }^{\circ} \mathrm{C}$. Adiponectin, leptin, tumor necrosis factor- $\alpha$ (TNF $\alpha)$, and MCP1 concentrations were determined using a Multiplex MAP Mouse Adipocyte Panel (Merck Millipore). 


\section{Preparation of cytoplasmic and nuclear fractions}

Cells were washed twice with ice-cold PBS, incubated at $4{ }^{\circ} \mathrm{C}$ for $5 \mathrm{~min}$ in hypotonic buffer $(10 \mathrm{mM}$ Tris- $\mathrm{HCl}$, $\mathrm{pH} 7.6,10 \mathrm{mM} \mathrm{NaCl}, 3 \mathrm{mM} \mathrm{MgCl}_{2}, 0.5 \% \mathrm{NP}-40$, and protease inhibitors), scraped from the dish, and centrifuged at $130 \mathrm{~g}$ for $5 \mathrm{~min}$. The supernatant was used as the cytoplasmic fraction. The pellet was resuspended in nuclear extraction buffer $(0.4 \mathrm{M} \mathrm{NaCl}, 5 \mathrm{mM}$ HEPES, $\mathrm{pH}$ 7.9, 26\% glycerol, $1.5 \mathrm{mM} \mathrm{MgCl}_{2}, 0.2 \mathrm{mM}$ EDTA, $0.5 \mathrm{mM}$ dithiothreitol, and protease inhibitors), incubated at $4{ }^{\circ} \mathrm{C}$ for $30 \mathrm{~min}$, and then centrifuged at $30,000 \mathrm{~g}$ for $20 \mathrm{~min}$. The supernatant resulting from this step was used as the nuclear fraction.

\section{Immunoblot analysis}

Cells were lysed in RIPA buffer and $30 \mu \mathrm{g}$ of each protein sample was separated using $10 \%$ SDSpolyacrylamide gel electrophoresis and transferred onto a nitrocellulose membrane (Schleicher \& Schuell, Dassel, Germany). The membranes were incubated with mouse anti-GAPDH (1:500; Santa Cruz Biotechnology, Dallas, TX, USA), goat anti-Lamin B (1:500; Santa Cruz), or rabbit antisera against XBP1 (1:200; BioLegend, San Diego, CA, USA), GLUT4 (1:500; Chemicon, Temecula, CA, USA), PPARY (1:500; Santa Cruz), phospho-insulin receptor (P-IR) (1:500; Cell Signaling, Danvers, MA, USA), IR (1:500; Cell Signaling), phospho-insulin receptor substrate 1 (P-IRS1) (1:500; Cell Signaling), IRS1 (1:500; Cell Signaling), phospho-AKT (P-AKT; 1:500; Epitomics, Burlingame, CA, USA), AKT (1:500; Epitomics), NFkB (1:500; Cell Signaling), phospho-JNK (P-JNK; 1:500; Cell Signaling), JNK (1:500; Cell Signaling), FGF21 (1:500; Cusabio, College Park, MD, USA), phospho-IRE1 $\alpha$ (P-IRE1 $\alpha$; 1:500; Novus Biologicals, Littleton, CO, USA), and IRE1 $\alpha$ (1:500; Novus Biologicals). Antibody binding was detected using horseradish peroxidase-conjugated anti-rabbit, anti-mouse (1:2,000; Sigma), or anti-goat IgG (1:2000; Santa Cruz), and the immunoreactive bands were visualized using the ECL method (Amersham Pharmacia, Buckinghamshire, UK). For statistical analysis, immunoreactivity was quantified by densitometry using a Gel Doc EZ System (Bio-Rad, Hercules, CA, USA).

\section{Chromatin immunoprecipitation assay}

Cells were cross-linked with formaldehyde and then sonicated to generate 200-1000 bp DNA fragments. The fragmented chromatin samples were immunoprecipitated with rabbit anti-mouse XBP1 (5 $\mu$ g; Santa Cruz), reverse cross-linked, purified, and analyzed by PCR according to the supplier's protocol (Merck Millipore). Primers for mouse $f g f 21$ promoter region amplicons are listed in Table 1. DNA samples that were immunoprecipitated with rabbit IgG were used as negative controls.

\section{Assays for XBP1 mRNA splicing}

To detect the activation of the IRE1/XBP1s pathway in mature adipocytes, we generated an XBP1-EGFP fusion reporter construct ( $\mathrm{pEGFP}-\mathrm{mXBP} 1$ ). The XBP1 coding sequence was amplified using reverse transcriptase (RT)PCR, digested with EcoRI and SalI enzymes, and then subcloned into a pEGFP-N1 vector (Invitrogen). Subsequently, the EGFP coding region was inserted into the putative IRE1 $\alpha$ splice site, giving rise to a fusion protein that lacks the XBP1 C-terminal region. EGFP is out of frame without IRE1 $\alpha$-mediated splicing, but is in frame with XBP1 after the 23-base stem-loop of XBP1 is eliminated by splicing. Cells were transfected with pEGFP-mXBP1 and fixed for fluorescence microscopy. The green fluorescent area and intensity was quantified with ImageJ.

Next, to measure functional XBP1s activity, we generated an expression construct in which full-length wildtype unspliced XBP1 (XBP1u) gene is inserted (pcDNAWT-mXBP1u). XBP1u was amplified by RT-PCR, digested with NotI and BamHI enzymes, and subcloned into pcDNA3.1 vector (Invitrogen). To generate the mutation construct (pcDNA-Mut-mXBP1u), the 4-base putative IRE1 $\alpha$ splicing site was mutagenized using a QuikChange Site-Directed Mutagenesis Kit (Stratagene, La Jolla, CA, USA) (EMM 2016 48:e226). These two constructs were transfected into cells by electroporation. To examined the IRE1 $\alpha$ spedicificty of XBP1 mRNA splicing, cells were pretreated with the IRE1 $\alpha$ inhibitor STF-083010 $(50 \mu \mathrm{M}$, Sigma) for $1 \mathrm{~h}$.

\section{PPARY activity assay}

The PPARY responsive element reporter was generated by inserting three copies of the DR1 sequence (TTCTGACCTATGACCTGG) into a pGL3-basic reporter plasmid (pGL3-DR1). Cells $\left(2 \times 10^{4}\right.$ per well in 24-well plates) were co-transfected with pGL3-DR1 (50 ng) and either siRNA $(20 \mathrm{nM})$ or plasmid $(500 \mathrm{ng})$. Cells were then treated with reagents including PPAR $\gamma$ activator (rosiglitazone, $1 \mu \mathrm{M})$ (Sigma) or inhibitor (GW9662, $1 \mu \mathrm{M})$ (Sigma), IRE1 $\alpha$ inhibitor STF-083010 $(50 \mu \mathrm{M})$, or palmitate $(200 \mu \mathrm{M})$ as indicated. After $48 \mathrm{~h}$, cells were lysed and luciferase activity was measured using a Dual Luciferase Assay Kit (Promega, Madison, WI, USA). Transfection efficiency was normalized using the renilla-expressing plasmid (pRL-CMV-luc; $5 \mathrm{ng}$ ).

\section{Statistical analysis}

All values are expressed as the means \pm SEM from at least three independent experiments. The data were analyzed using Student's $t$-test or one- or two-way analysis of variance followed by Tukey's multiple comparison test. Statistical analyses were performed using GraphPad Prism 5 for Windows (GraphPad Prism Software, La Jolla, 
CA, USA). In all cases, statistical significance was accepted at $P<0.05$.

\section{Results \\ XBP1s enhances insulin-stimulated glucose uptake in adipocytes}

The major physiological action of insulin on glucose homeostasis is to stimulate the cellular uptake of glucose in insulin-sensitive organs, such as skeletal muscle and adipose tissue. To examine the role of XBP1s in insulin sensitivity in adipocytes, we first measured the rate of insulin-dependent glucose uptake in XBP1soverexpressing mouse adipocytes (Fig. 1a). In the absence of insulin, XBP1s did not increase the glucose uptake rate; however, in adipocytes treated with insulin (1 $\left.\mu \mathrm{g} \mathrm{ml}^{-1}, 30 \mathrm{~min}\right) \mathrm{XBP} 1 \mathrm{~s}$ significantly increased the glucose uptake rate. Additionally, glucose uptake was not reduced upon XBP1s knockdown regardless of the presence of insulin (Fig. 1b), indicating that XBP1s is expressed at very low levels in mature adipocytes ${ }^{16}$.

Next, we examined the effect of XBP1s on the expression of GLUT4, the principal insulin-sensitive glucose transporter in adipocytes ${ }^{17,18}$. Although XBP1s is a functionally active transcription factor, XBP1s overexpression did not increase GLUT4 expression at either the mRNA or protein levels, indicating that GLUT4 is not an XBP1s target gene (Fig. 1c, left). However, in the presence of insulin, XBP1s overexpression clearly increased GLUT4 translocation from the cytoplasm to the cell surface (Fig. 1c, right). It is well known that GLUT4 redistribution is regulated by insulin receptor signaling pathways; ${ }^{17,18}$ therefore, we examined whether XBP1s is involved in the activation of insulin signaling in adipocytes. As shown in Fig. 1d, XBP1s significantly elevated insulin receptor-mediated phosphorylation of IRS1 and AKT (Fig. 1d) in insulin-treated adipocytes, suggesting that XBP1s amplifies insulin signaling in adipocytes. Taken together, these findings implicate XBP1s as a putative positive regulator of insulin sensitivity in mature adipocytes.

\section{XBP1s increases insulin-mediated PPAR $\gamma$ activity in adipocytes}

Adipose tissue is the major mediator through which PPARY affects insulin sensitivity. Activation of adipose PPAR $\gamma$ induces the expression of several genes encoding proteins involved in the insulin signaling cascade, thereby increasing insulin-mediated glucose uptake and regulating the release of adipokines ${ }^{19}$. In our previous reports, we indicated that XBP1s is a principal regulator of PPAR $\gamma$ transcription during adipogenesis ${ }^{8-10}$. Accordingly, we first examined the effect of XBP1s on PPAR $\gamma$ expression in mature adipocytes. However, in contrast to its effect in preadipocytes, XBP1s overexpression did not increase
PPAR $\gamma$ expression at either the mRNA (total and PPAR 2 isoforms) or protein (PPAR 1 and PPAR 2 ) levels (Fig. 2a). In contrast, XBP1s overexpression significantly increased PPAR $\gamma$ activity by approximately 2.5-fold. Treatment of adipocytes with the PPAR $\gamma$ antagonist GW9662 almost completely suppressed XBP1s-induced PPAR $\gamma$ activity and mRNA levels of typical PPAR $\gamma$ target genes such as Ap2 and CD36 (Fig. 2b). The effect of XBP1s on insulin-stimulated glucose uptake was also significantly decreased upon GW9662 treatment (Fig. 2c). In addition, XBP1s increased the expression and secretion of adiponectin (Fig. 2d), an adipokine that is a known target of PPAR $\gamma^{20}$ and a well-known insulin-promoting protein that facilitates glucose uptake through increased GLUT4 translocation ${ }^{21}$. These results strongly suggest that the functional activation of endogenous PPAR $\gamma$, and the resulting increase in adiponectin production, may be the principal mechanism by which XBP1s mediates increased cell surface translocation of GLUT4 and glucose uptake in mature adipocytes.

\section{FGF21 is a key regulator of XBP1s-mediated PPARY activation in adipocytes}

As XBP1s is an active transcription factor, it can be easily inferred that other mediators that are transcriptionally induced by XBP1s may be involved in insulin-stimulated PPAR $\gamma$ activation in adipocytes. Among the known PPAR $\gamma$ activating proteins, FGF21 has been reported to be induced by XBP1s in hepatocytes ${ }^{22}$ and is suggested to be a potent activator of glucose uptake in adipose tissue $\mathrm{e}^{23-25}$. We thus examined FGF21 as a putative target of XBP1s in the regulation of insulin sensitivity in mature adipocytes. XBP1s significantly increased the mRNA and protein levels of FGF21 only in the presence of insulin (Fig. 3a), and increased XBP1s enrichment was observed in the $f g f 21$ promoter region of insulin-treated adipocytes (Fig. 3b). Moreover, XBP1sinduced regulation of PPAR $\gamma$, target gene expression (Fig. 3c), and glucose uptake (Fig. 3d) were significantly suppressed by FGF21 knockdown, suggesting that XBP1smediated transcriptional activation of FGF21 may play an important role in insulin-stimulated PPAR $\gamma$ activation and glucose uptake in adipocytes.

\section{Insulin upregulates the generation and nuclear translocation of XBP1s in adipocytes}

Because our results (Fig. 1) and previous report ${ }^{16}$ showed that the basal level of XBP1s was too low to activate PPARY activity, we postulated that insulin signaling might increase XBP1s levels in mature adipocytes. As shown in Fig. 4a, treatment with insulin $(6 \mathrm{~h})$ increased XBP1s mRNA levels in a dose-dependent manner. In time-course experiments, insulin $\left(1 \mu \mathrm{g} \mathrm{ml}^{-1}\right)$ induced XBPls transcription both rapidly and transiently; XBP1s 

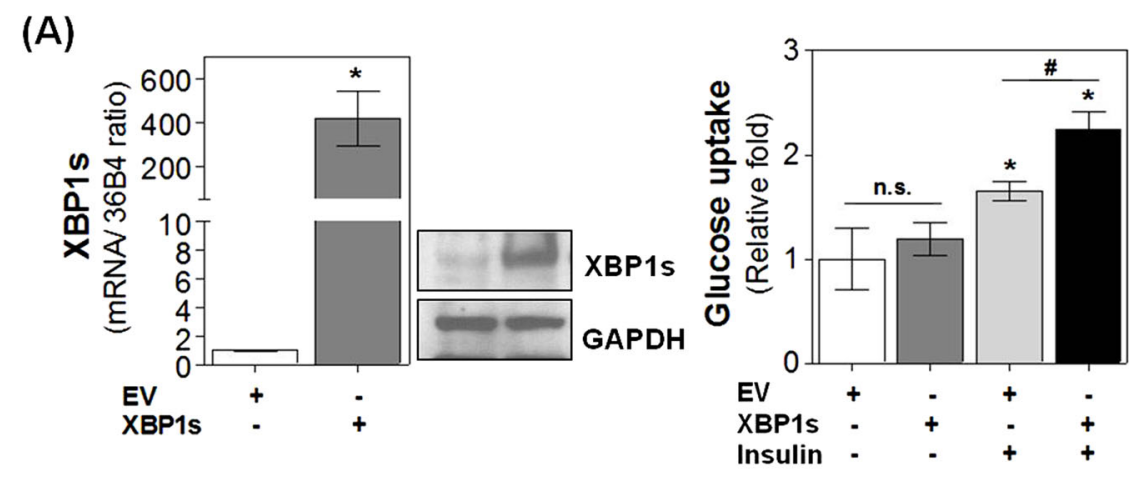

(B)
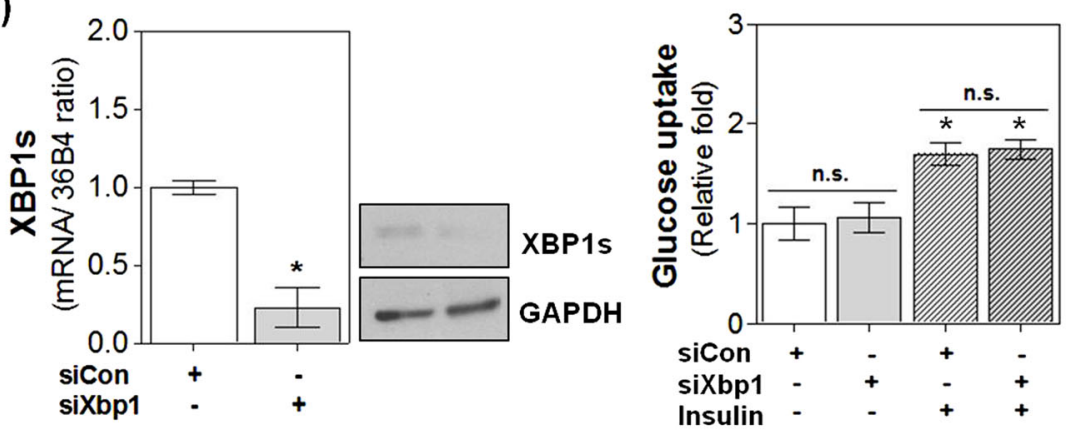

(C)
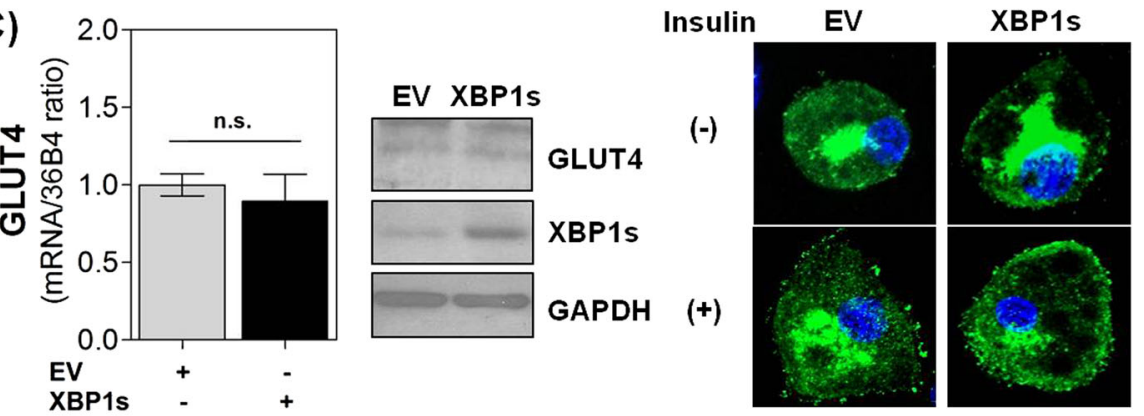

GLUT4 \& DAPI

(D)
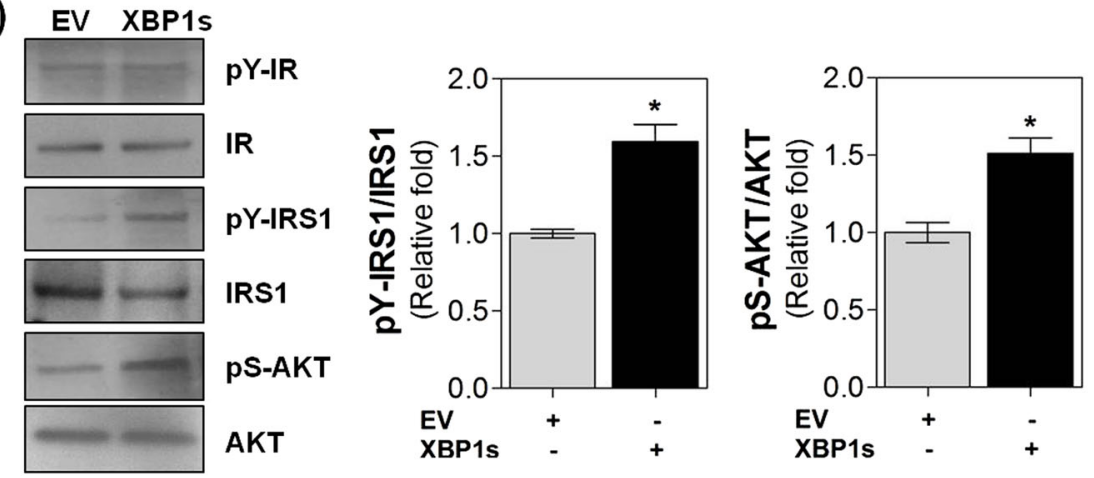

Fig. 1 Effect of XBP1s on insulin-stimulated glucose uptake in 3T3-L1 adipocytes. 3T3-L1 adipocytes were transfected with pCDNA3.1-mXBP1s (XBP1s) or an siRNA against XBP1 (siXBP1), and treated with insulin $\left(1 \mu \mathrm{g} \mathrm{m}{ }^{-1}\right)$ for $30 \mathrm{~min}$ as indicated. $\mathbf{a}, \mathbf{b}$ Intracellular XBP1s mRNA and protein levels and glucose uptake rates were determined. c The expression (left) and translocation (right, green) of GLUT4 were analyzed using real-time PCR/ immunoblot analyses and immunofluorescence, respectively. DAPI was used to stain nuclei (right, blue). d The total and phosphorylated expression levels of insulin receptor (IR), insulin receptor substrate 1 (IRS1), and AKT were examined by immunoblot analysis (left) and quantified with densitometry (right). Values are presented as the means \pm SEM of three independent experiments. ${ }^{*} P<0.05$ compared to empty vector (EV) or control siRNA (siCon); ${ }^{P} P<0.05$ compared to insulin-treated EV. n.s. not significant 

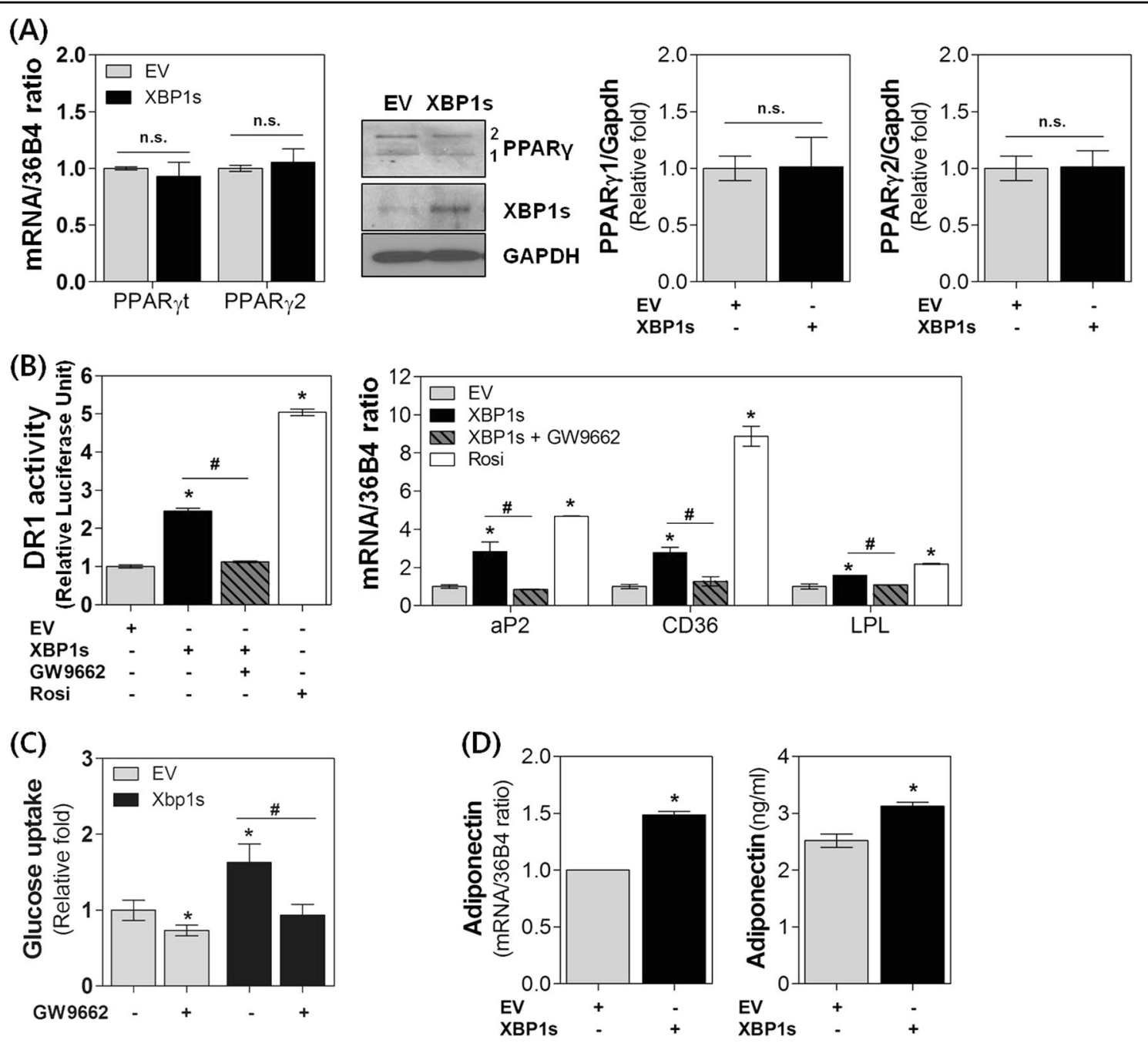

Fig. 2 XBP1s enhances insulin-mediated PPARY activation in adipocytes. 3T3-L1 adipocytes were transfected with control vector (EV) or pcDNA3.1-mXBP1s (XBP1s) and treated for $30 \mathrm{~min}$ with insulin $\left(1 \mu \mathrm{g} \mathrm{ml} \mathrm{l}^{-1}\right)$. a XBP1s and PPARY (1 and 2, or total) mRNA and protein levels were analyzed by real-time PCR and/or immunoblotting (left) and quantified with densitometry (right). $\mathbf{b}$ The effect of an XBP1s and PPARY activator (rosiglitazone, Rosi) or inhibitor (GW9662) on DR1 promoter activity (left) and the expression of the PPAR $\boldsymbol{y}$ target genes aP2, CD36, and LPL (right) were examined by real-time PCR. c The effect of GW9662 on the XBP1s-mediated increase in glucose uptake was measured. $\mathbf{d}$ The effect of XBP1s on adiponectin mRNA and secreted protein levels were determined using real-time PCR and ELISA, respectively. Values are presented as the means \pm SEM of three independent experiments. ${ }^{*} P<0.05$ compared to EV. ${ }^{\#} P<0.05$ compared to XBP1s. n.s. not significant

mRNA levels increased beginning at $1.5 \mathrm{~h}$, peaked at $6 \mathrm{~h}$ (3.3-fold increase), and returned to control levels by $24 \mathrm{~h}$ (Fig. 4b). XBP1s is produced by unconventional splicing of the XBP1 mRNA. To verify the involvement of insulin in XBP1 mRNA splicing, we examined the effect of insulin on the phosphorylation of IRE1 $\alpha$, an exclusive inducer of $\mathrm{XBP} 1$ posttranscriptional splicing. As shown in Fig. 4c, insulin strongly induced IRE1 $\alpha$ phosphorylation as well as the expression of both XBP1s and FGF21 in a dosedependent manner, suggesting that the specific activation of IRE1 $\alpha$ is important for insulin-induced XBP1s production. Additionally, insulin increased total (unspliced plus spliced) levels of XBP1 mRNA $6 \mathrm{~h}$ after insulin treatment (Fig. 4b right), though this effect was much less potent than the effect of insulin on XBP1s levels. Correspondingly, the expression of ATF6 $\alpha$, a transcription factor responsible for the production of XBP1s, was also increased by insulin with the same temporal pattern (Fig. 4c). Taken together, these findings suggest that insulin may increase XBP1s mRNA levels principally by stimulating IRE1 $\alpha$-mediated splicing but not by stimulation XBP1 transcription.

With regard to the effect of insulin on XBP1s, recent reports have suggested that insulin promotes the nuclear translocation of XBP1s, thus increasing its transcriptional activity in kidney podocytes and the liver ${ }^{12,26}$. In our 


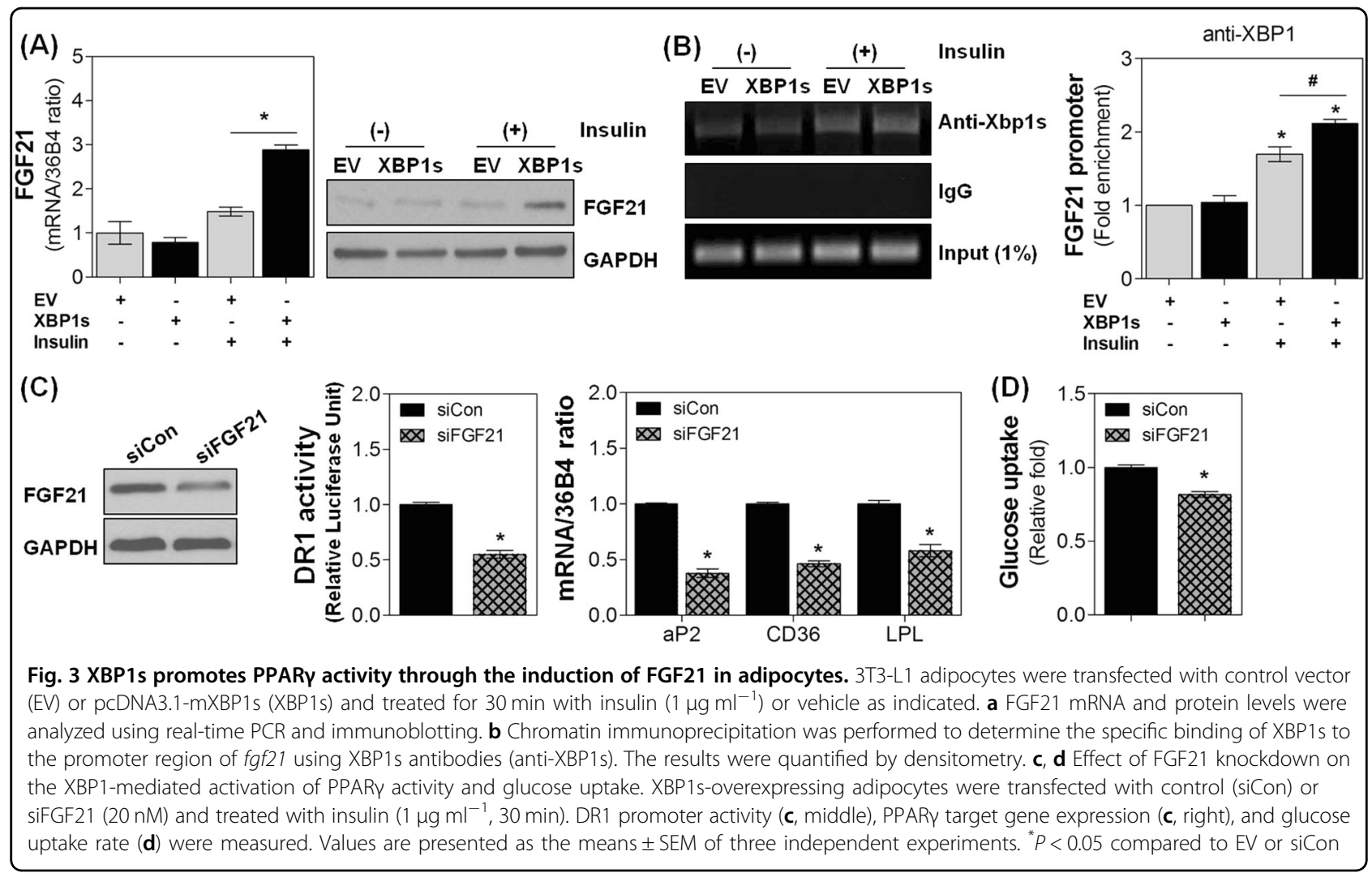

experiments using XBP1s-overexpressing adipocytes, insulin $\left(1 \mu \mathrm{g} \mathrm{ml}^{-1}, 30 \mathrm{~min}\right)$ significantly increased XBP1s nuclear translocation (Fig. 4d) as well as the expression of two target genes, ERdj4 and p58 ${ }^{\mathrm{IPK}}$ (Fig. 4e). In addition, the insulin-mediated increase in FGF21 expression in wild-type adipocytes was significantly reduced by XBP1s knockdown (Fig. 4f). Taken together, these findings indicate the functional importance of insulin-induced generation and activation of XBP1s in adipocytes.

\section{Physiological importance of insulin-mediated XBP1 mRNA splicing}

IRE1 $\alpha$ catalyzes unconventional mRNA splicing by removing an internal 26 nucleotides from the XBP1 mRNA. To confirm the involvement of IRE1 $\alpha$ in the insulin-mediated increase in XBP1s production, we generated an XBP1-EGFP fusion construct designed to express EGFP only after the 26-base stem-loop of the XBP1 mRNA was spliced out by IRE1 $\alpha$ endonuclease activity (Fig. 5a; also see Materials and methods). We then analyzed expression of the EGFP epitope upon insulin treatment. As shown in Fig. 5b, insulin treatment $\left(1 \mu \mathrm{g} \mathrm{ml}^{-1}, 24 \mathrm{~h}\right)$ significantly increased the area and the intensity of both EGFP expression (Fig. 5b, d) and XBP1s mRNA production (Fig. $5 \mathrm{c}$ ). These findings indicate that
IRE1 $\alpha$ activation is a critical mechanism underlying insulin-induced XBP1s production.

To further examine the functional importance of insulin-stimulated splicing of mRNA through IRE1 $\alpha$ activation, we developed the pcDNA-WT-mXBP1u construct in which the wild-type XBP1u gene was inserted. Thus, functional XBP1s protein could be produced only upon IRE $1 \alpha$ activation (Fig. 5e upper; WT). In adipocytes transfected with pcDNA-WT-mXBP1u, both mRNA and protein XBP1s levels were significantly increased by insulin treatment (Fig. 5f), indicating the establishment of an insulin-mediated splicing system in adipocytes. In these cells, insulin $\left(1 \mu \mathrm{g} \mathrm{ml}^{-1}, 6 \mathrm{~h}\right)$ significantly increased FGF21 mRNA levels (Fig. 5g), DR1 activity (Fig. 5h), and glucose uptake (Fig. 5i). These insulin-mediated effects were completely reversed upon pretreatment with the IRE1 $\alpha$ inhibitor STF-083010 (50 uM, $1 \mathrm{~h}$ ). In addition, insulin-mediated effects almost disappeared in adipocytes transfected with a mutated construct, pcDNA-MutmXBP1u, in which functional XBP1s protein cannot be generated due to a 12 nt mutation in the IRE1 $\alpha$ recognizing site within the XBP1u mRNA (Fig. 5e, lower; Mut) (Fig. 5j-1). These findings implicate that insulin-mediated IRE1 $\alpha$ activation in adipocytes is a critical step in the production of functional XBP1s as well as 


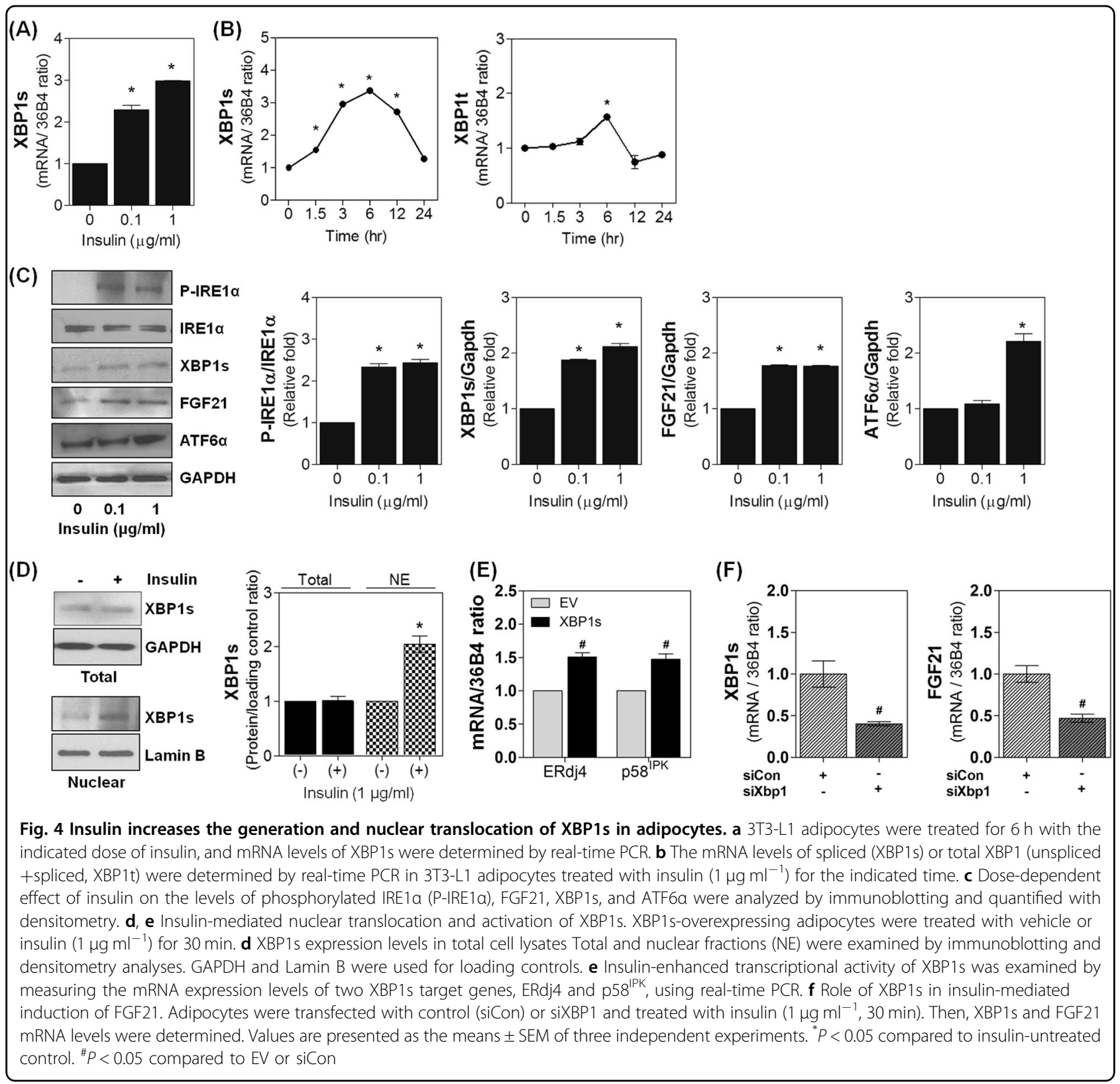

the subsequent increases in FGF21 levels, PPAR $\gamma$ activity, and glucose uptake.

\section{$\mathrm{XBP} 1 \mathrm{~s}$ attenuates palmitate-induced insulin resistance in adipocytes}

Previous studies have shown that rosiglitazone, a potent PPAR $\gamma$ agonist, ameliorates insulin resistance in adipocytes $^{27}$. Based on our findings demonstrating PPAR $\gamma$ activation by XBP1s, we investigated whether XBP1s could improve insulin resistance induced by treating adipocytes with palmitate $(200 \mu \mathrm{M}, 24 \mathrm{~h})$, a well-known in vitro model for studying insulin resistance ${ }^{28}$. As shown in Fig. 6a, palmitate significantly inhibited insulin signaling by suppressing the levels of phosphorylated IRS1 and AKT. Insulin-mediated effects on GLUT4 cell surface translocation (Fig. 6b) and subsequent glucose uptake mostly disappeared in palmitate-treated adipocytes (Fig. 6c), indicating the establishment of a reliable in vitro insulin resistance model in adipocytes. However, upon palmitate treatment of XBP1s-overexpressing cells, the palmitate-induced suppression of insulin signaling and glucose uptake in adipocytes was significantly reversed (Fig. 6a-c).

PPAR $\gamma$ activation also exerts systemic insulinsensitizing effects by regulating the expression of several genes involved in the synthesis of adipokines from adipose 
(A)

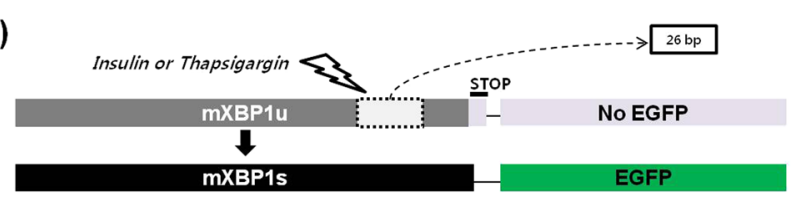

(B)
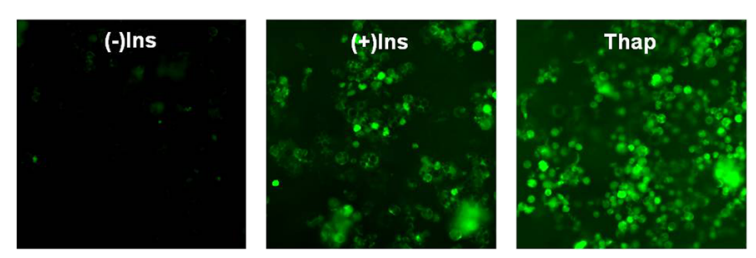

(C)

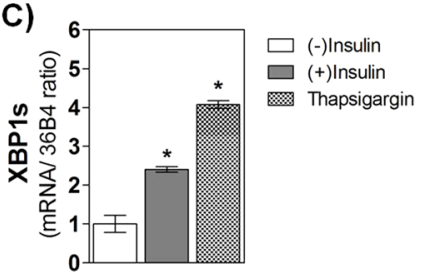

(E)

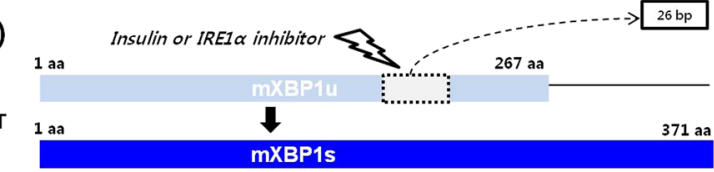

WT

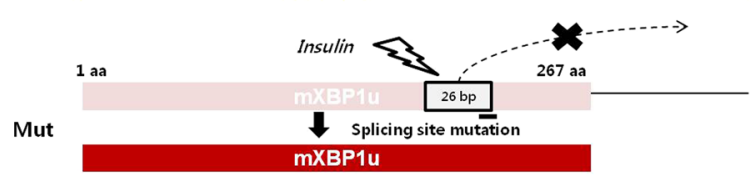

(G)

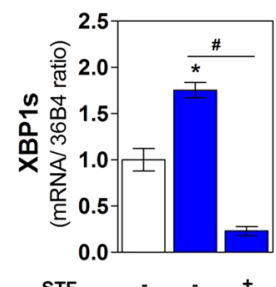

STF : ;

(J)
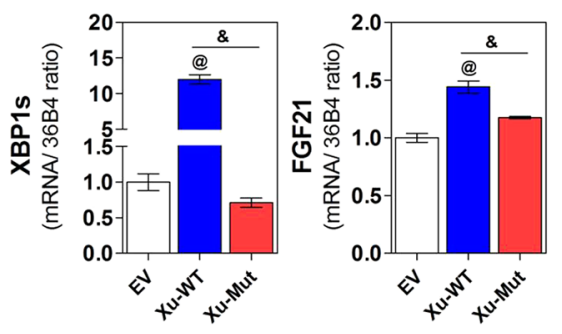

(D)

(K)

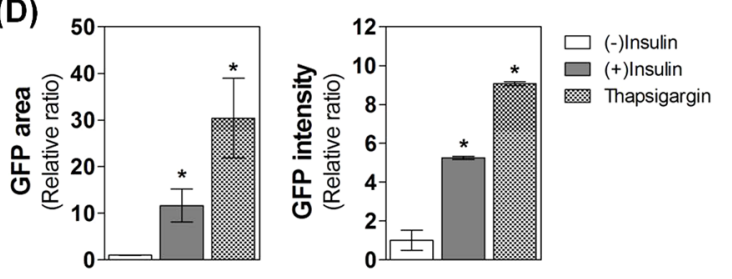

(F)

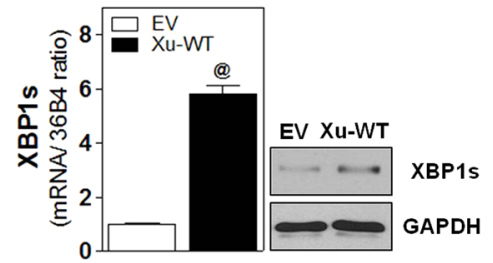

(I)

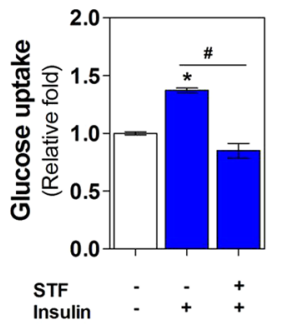

(L)
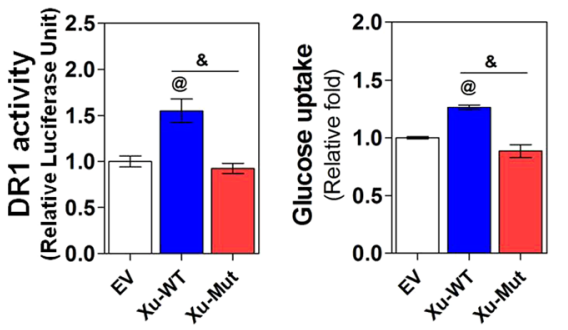

Fig. 5 (See legend on next page.) 
(see figure on previous page)

Fig. 5 IRE1a-mediated XBP1 mRNA splicing is critical for insulin-stimulated glucose uptake in adipocytes. a Diagram of the pEGFP-mXBP1 reporter construct (see Materials and methods). b-d 3T3-L1 adipocytes were transfected with pEGFP-mXBP1 and treated for $6 \mathrm{~h}$ with insulin (1 $\mu \mathrm{g}$ $\mathrm{ml}^{-1}$ ) or thapsigargin (200 nM). The levels of GFP protein (b) and XBP1s mRNA (c) were examined using fluorescence microscopy and real-time PCR, respectively. GFP expression area and intensity were quantified with ImageJ (d). e Diagram of wild-type (pcDNA-WT-mXBP1u, upper) or mutant (pcDNA-Mut-mXBP1u, lower) constructs used for functional analyses of IRE1a-induced XBP1 splicing (see Materials and methods). $\mathbf{f}-\mathbf{i}$ Adipocytes were transfected with pcDNA-WT-mXBP1u construct, pretreated for $1 \mathrm{~h}$ with vehicle or IRE1a inhibitor STF-083010 (STF, $50 \mathrm{uM})$, then treated with insulin $\left(1 \mu \mathrm{g} \mathrm{ml}^{-1}, 6 \mathrm{~h}\right)$ as indicated. $\mathbf{f}$ Effect of insulin on XBP1s mRNA and protein levels in adipocytes transfected with pcDNA-WT-mXBP1u. $\mathbf{g}-\mathbf{i}$ Effect of STF-08310 on XBP1s and FGF21 mRNA levels, DR1 activity, and glucose uptake rate. $\mathbf{j}-\mathbf{I}$ Effect of mutant construct (pcDNA-Mut-mXBP1u) on XBP1s and FGF21 mRNA levels, DR1 activity and glucose uptake rate. Values are presented as the means \pm SEM of three independent experiments. ${ }^{*} P$ $<0.05$ compared to the insulin-untreated group. ${ }^{\#} P<0.05$ compared to the insulin-treated group. ${ }^{@} P<0.05$ compared to the EV-transfected group. ${ }^{\&} P<0.05$ compared to the pCDNA-WT-mXBP1u-transfected group

tissue $^{4}$. As expected, intracellular mRNA and secreted protein levels of adipokines were altered in palmitatetreated adipocytes in our study. Namely, we observed a decrease in adiponectin and an increase in proinflammatory cytokines including leptin, MCP1 and TNF $\alpha$ (Fig. 6d, e). In accordance with these findings, expression levels of NFKB and P-JNK, two major signaling molecules that stimulate inflammation in adipocytes ${ }^{29}$, were also significantly increased upon palmitate treatment (Fig. 6f). In addition, glycerol release from adipocytes was significantly increased upon palmitate treatment, indicating that palmitate stimulates adipocyte lipolysis (Fig. 6g). However, just as we have observed above, ectopic XBP1s expression significantly reversed palmitateinduced dysregulation of adipokine expression and release (Fig. 6d, e), NFKB and P-JNK expression (Fig. 6f), and lipolysis (Fig. 6g). These findings strongly suggest that $\mathrm{XBP} 1 \mathrm{~s}$ has the potential to attenuate or reverse pathophysiological phenotypes associated with insulin resistance both in adipocytes and at the whole-body level.

\section{Discussion}

In this study, we provide the first evidence that XBP1s is produced by insulin signaling and is an active mediator of insulin-stimulated glucose uptake in adipocytes through its effects on PPAR $\gamma$ activity. The effect of XBP1s on glucose uptake was completely abolished by a PPAR $\gamma$ inhibitor, suggesting that PPAR $\gamma$ activation is the exclusive mechanism by which XBP1s induces insulin sensitization in adipocytes. Of note, XBP1s regulation of PPAR $\gamma$ activity and the subsequent increase in glucose uptake by adipocytes were insulin-dependent; XBP1s-mediated increases in glucose uptake, GLUT4 translocation, PPAR $\gamma$ activity, and FGF21 expression were observed only in the presence of insulin. As GLUT4 translocation and glucose uptake in adipocytes are known to be regulated by PPAR $\gamma$, these findings indicate that insulin signaling is necessary for XBP1s-induced PPAR $\gamma$ activation. Based both on our data and the present state of knowledge within the field, we propose a few possible mechanisms for this observation. First, increasing evidence demonstrates possible interactions between insulin signaling pathways and XBP1s. For example, XBP1s activates the phosphorylation of AKT, a key player in insulin-induced GLUT4 translocation in adipocytes ${ }^{18,30}$. Our results demonstrated that XBP1s increased the phosphorylation of both AKT and IRS1 in both insulin-sensitive and -resistant adipocytes. A recent report showing IRS1mediated regulation of XBP1s protein stability in pancreatic $\beta$ cells supports this assumption ${ }^{31}$. Second, insulin enhances XBP1s transcriptional activity by increasing its nuclear translocation in the livers of ob/ob mice ${ }^{11}$ and concordantly increases nuclear translocation and XBP1s target gene expression in adipocytes in our results. Third, insulin increases XBP1 mRNA splicing in adipocytes. Using two reporter assays that were specifically designed to directly measure XBP1 mRNA splicing and function, we clearly provided the first evidence that insulin enhances the generation of functionally active XBP1s through the phosphorylation of IRE1 $\alpha$. We also observed nearly complete abolition of the insulin-mediated effects on XBP1s, FGF21, PPAR $\gamma$, and glucose uptake upon treatment with an IRE1 $\alpha$ inhibitor, further supporting our conclusion. Considering that the levels of XBP1s are very low in the absence of insulin, these results imply that inducing the generation of XBP1s may be an indispensable additional step for insulin-mediated glucose uptake. Taken together, these findings strongly indicate that insulin sensitivity is maintained or amplified by a positive feedback mechanism involving XBP1s and insulin signaling molecules. Because XBP1s is ubiquitously expressed in our body, it would be interesting to investigate the role of XBP1s in insulin-sensitive tissues other than adipose tissue.

How does XBP1s increase PPARy activity in response to insulin signaling? Currently, many molecules and mechanisms promoting PPAR $\gamma$ activity have been suggested $^{32}$. Of these, we chose to investigate FGF21 because it was recently reported to be induced by XBP1s in the liver $^{22}$. FGF21 is an inducible factor secreted mainly from 


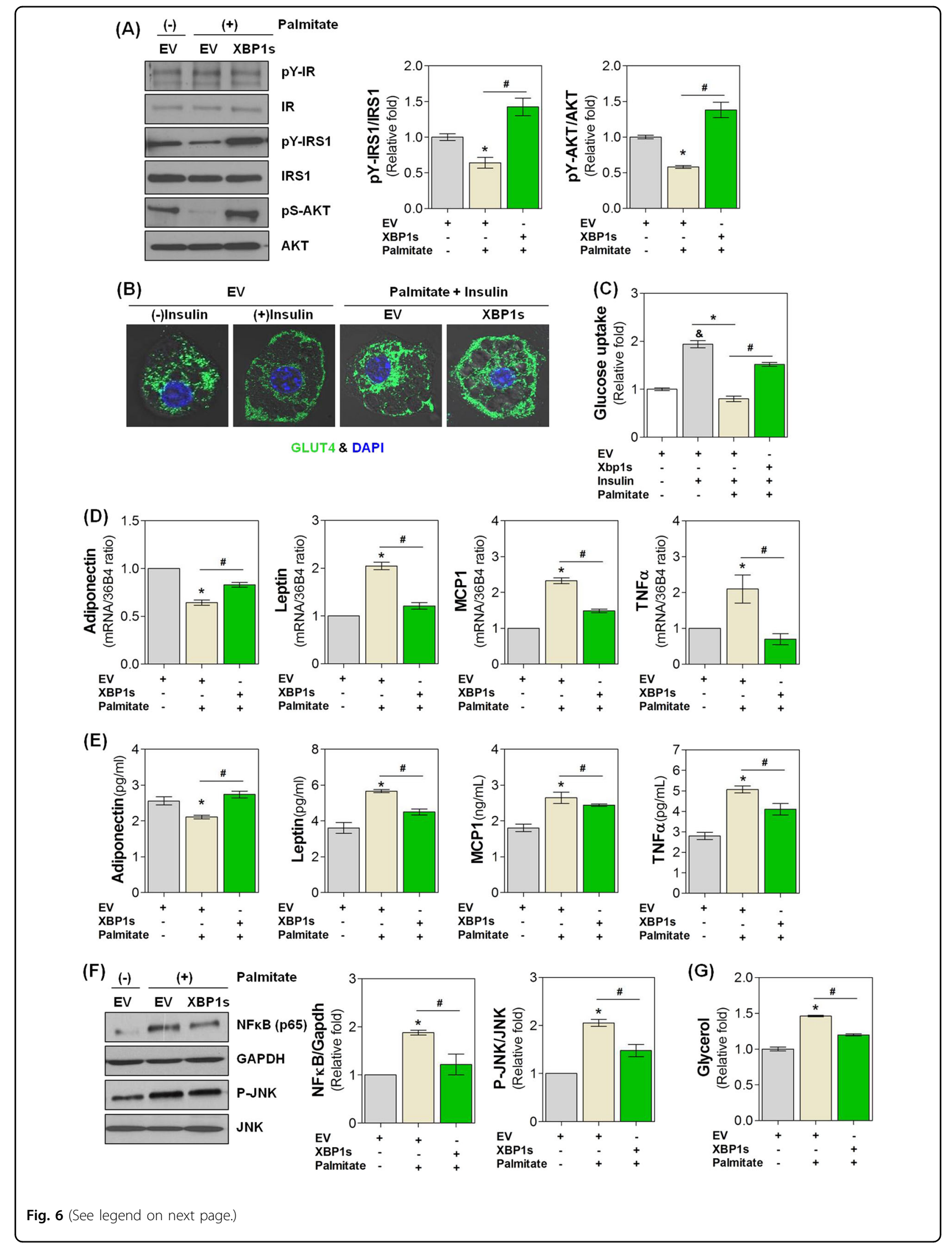


(see figure on previous page)

Fig. 6 XBP1s attenuates palmitate-induced insulin resistance in adipocytes. Adipocytes were transfected with control (EV) or pcDNA3.1-mXBP1s (XBP1s) and treated with vehicle or palmitate $(200 \mu \mathrm{M})$ for $24 \mathrm{~h}$. Cells were further incubated with insulin $\left(1 \mu \mathrm{g} \mathrm{ml}{ }^{-1}, 30\right.$ min) as indicated, and then the following parameters of insulin resistance were determined (see Materials and methods): a Insulin signaling; b GLUT4 distribution; c glucose uptake rate; $\mathbf{d}$, e Intracellular mRNA and secreted protein levels of adipokines such as adiponectin, leptin, MCP1, and TNFa; $\mathbf{f}$ NFKB and P-JNK expression; and $\mathbf{g}$ glycerol release rate. Values are presented as the means \pm SEM of three independent experiments. ${ }^{*} P<0.05$ compared to EV control. ${ }^{\#} P<0.05$ compared to palmitate control. ${ }^{\circledR} P<0.05$ compared to insulin-untreated and palmitate-untreated group

the liver, adipose tissue, and skeletal muscle that exerts tissue-specific functions. In adipose tissue, FGF21 acts in a paracrine manner to promote insulin sensitivity by amplifying PPARy activity ${ }^{24}$. A possible reciprocal mechanism by which PPAR increases FGF21 expression in adipose tissue has also been reported ${ }^{33}$. However, the mechanism underlying this process remains unknown. Recently, it has been suggested that functional insulin signaling in adipose tissue is required for FGF21-mediated sensitization to insulin ${ }^{25}$, indicating the possibility that XBP1s may link insulin signaling and PPAR $\gamma$ activation by increasing FGF21 levels in adipocytes. In our study, we present the first evidence that XBP1s strongly amplifies the expression of FGF21, which plays a role in the insulininduced activation of PPAR $\gamma$ in insulin-treated adipocytes. To the best of our knowledge, FGF21 does not affect the transcription of PPAR $\gamma$; therefore, these findings indicate that the induction of FGF21 and the potential crosstalk between FGF21 and PPAR $\gamma$ is the main mechanism by which XBP1s mediates insulin-stimulated PPAR $\gamma$ activation and glucose uptake in adipocytes.

Saturated free fatty acids, such as palmitate, provoke inflammatory responses and cause insulin resistance in adipocytes through dysregulation of $\mathrm{PPAR}^{34}$. In addition, PPARy activation in adipose tissue and the resulting modulation of pro- and anti-inflammatory mediators has been widely accepted as an essential mechanism by which both glucose uptake and systemic insulin sensitivity can be improved ${ }^{35,36}$. Here, we have shown evidence that $\mathrm{XBP} 1 \mathrm{~s}$ alleviates palmitate-induced insulin resistance in adipocytes. XBP1s potently reversed palmitate-induced suppression of insulin signaling and dysregulation of adipokine expression, resulting in an increase in both the translocation of GLUT4 to the cell surface and glucose uptake in insulin-resistant adipocytes. In particular, XBP1s increased adiponectin, a well-recognized anti-diabetic adipokine, in both insulin-sensitive and -resistant cells. In this regard, Sha et al. recently reported that XBP1s promotes multimerization of adiponectin to form the most potent adiponectin isotype ${ }^{15}$. In addition, we also demonstrated that XBP1s strongly reduced fatty acid release from palmitate-treated adipocytes. To maintain systemic insulin sensitivity, it is important to reduce fatty acid release into the blood and the subsequent aberrant accumulation of lipids in non-adipose tissues. Considering that previous reports have demonstrated anti-lipolytic activities of FGF21 and PPAR $\gamma^{37,38}$, two downstream targets of XBP1s in our study, these findings suggest that the suppression of lipolysis is an additional potential mechanism of XBP1s-induced regulation of insulin resistance. The promotion of adipogenesis is an additional mechanism by which systemic insulin sensitivity is maintained; this process leads to a greater number of smaller adipocytes with increased lipid storage capacity and adiponectin production. Further, PPAR $\gamma$ has been widely accepted as a master regulator of adipogenesis. We have previously reported a few mechanisms by which $\mathrm{XBP} 1 \mathrm{~s}$ stimulates adipogenesis through increasing PPARY expression in preadipocytes, implying that XBP1s may positively regulate systemic insulin sensitivity by increasing adipose tissue mass. In addition, PPAR $\gamma$ is suggested to regulate the inflammatory potential of adipose tissue macrophages, a central player in obesity-associated systemic insulin resistance ${ }^{39}$. Accordingly, PPAR $\gamma$ deletion or inactivation prevented anti-inflammatory polarization of macrophages and thus resulted in insulin resistance and glucose intolerance ${ }^{39,40}$. Interestingly, adipokines that were regulated by the XBP1s-PPARY axis in our data, such as MCP1, TNF $\alpha$, and leptin, are well-known factors regulating macrophage polarization ${ }^{40,41}$, indicating that $\mathrm{XBP} 1 \mathrm{~s}$ may regulate adipose tissue macrophages thorough a paracrine mechanism. Moreover, in our findings, XBP1s strongly suppressed the palmitate-induced phosphorylation of JNK and $\mathrm{NFK}_{\mathrm{K}} \mathrm{B}$, two central signaling molecules driving M1 macrophage polarization ${ }^{42,43}$. Although we did not examine the role of XBP1s in macrophages in this study, it seems likely that XBP1s may play an antiinflammatory role in this cell type. Taken together, these findings suggest that adipose XBP1s may control systemic glucose homeostasis and insulin sensitivity via a complex network of signaling pathways modulating adipokine secretion, lipolysis, adipogenesis, and the possible crosstalk between adipocytes and macrophages in adipose tissue.

In summary, XBP1s is generated and transcriptionally activated by insulin-mediated activation and nuclear translocation of IRE1 $\alpha$, thereby enhancing insulinstimulated glucose uptake in mature adipocytes through 


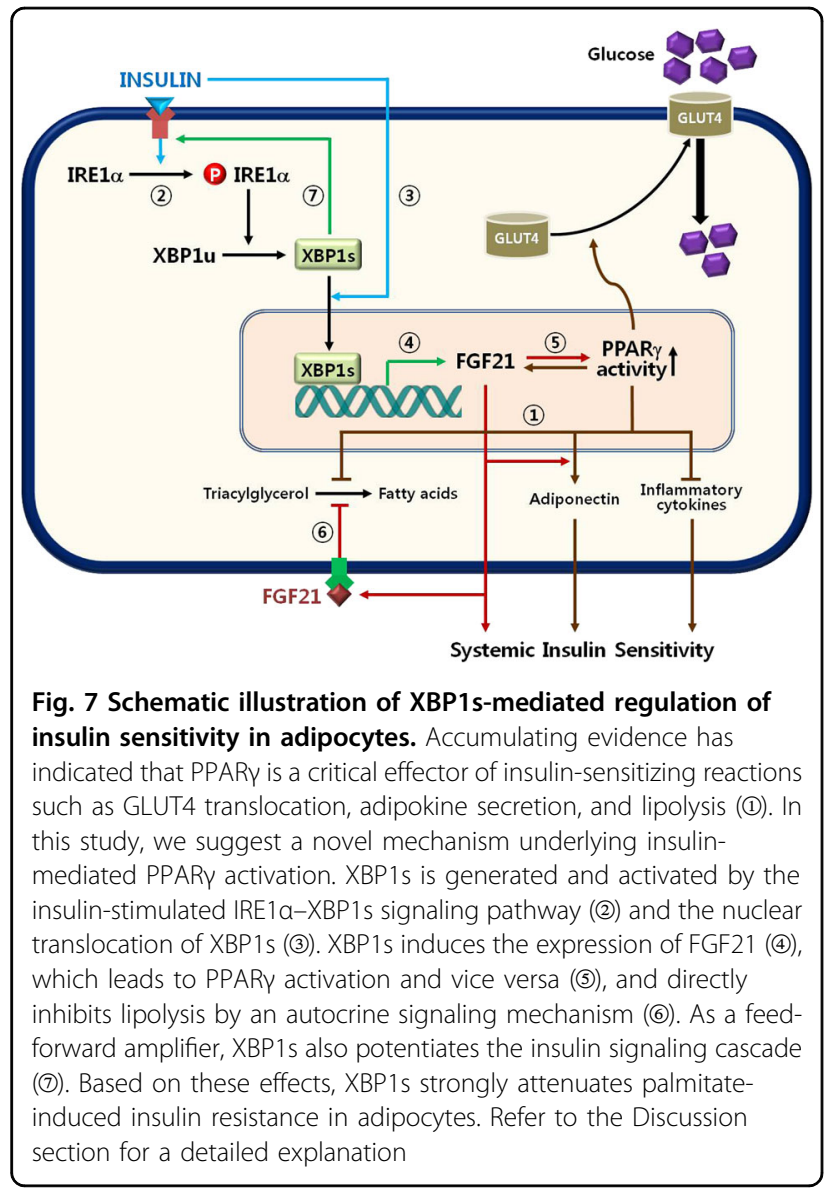

a mechanism involving FGF21 induction and subsequent PPARY activation (Fig. 7). XBP1s also regulates adipokine secretion and lipolysis in palmitate-treated adipocytes, suggesting a potential role for alleviating insulin resistance. When we combine these findings with the putative roles of XBP1s obtained from previous reports, it is very plausible that XBP1s is a critical factor for regulating insulin sensitivity in adipose tissue as well as at the wholebody level by enhancing PPAR $\gamma$ activity and/or expression.

\section{Acknowledgements}

This research was supported by the Basic Science Research Program through the National Research Foundation of Korea (NRF) funded by the Ministry of Education (2017R1D1A1B03031063) in 2017. The authors wish to acknowledge the financial support of the Catholic Medical Center Research Foundation during the 2018 program year.

\section{Author contributions}

The work presented here was carried out in collaboration between all authors. O.J.K. and Y.M.C. defined the research theme, interpreted the results, and prepared the manuscript. Y.M.C. and D.H.K. carried out the laboratory experiments and analyzed the data. K.H.L. and S.-W.J. discussed data analysis and interpretation.

\section{Conflict of interest}

The authors declare that they have no conflict of interest.

\section{Publisher's note}

Springer Nature remains neutral with regard to jurisdictional claims in published maps and institutional affiliations.

Received: 19 January 2018 Revised: 9 April 2018 Accepted: 20 April 2018. Published online: 15 August 2018

\section{References}

1. Vegiopoulos, A., Rohm, M. \& Herzig, S. Adipose tissue: between the extremes. EMBO J. 36, 1999-2017 (2017).

2. Czech, M. P. Insulin action and resistance in obesity and type 2 diabetes. Nat. Med. 23, 804-814 (2017).

3. Rieusset, J. et al. Insulin acutely regulates the expression of the peroxisome proliferator-activated receptor-gamma in human adipocytes. Diabetes $\mathbf{4 8}$ 699-705 (1999).

4. Leonardini, A., Laviola, L., Perrini, S., Natalicchio, A. \& Giorgino, F. Cross-talk between PPARgamma and insulin signaling and modulation of insulin sensitivity. PPAR Res. 2009, 818945 (2009).

5. Gustafson, B., Hedjazifar, S., Gogg, S., Hammarstedt, A. \& Smith, U. Insulin resistance and impaired adipogenesis. Trends Endocrinol. Metab. 26, 193-200 (2015).

6. Kroker, A. J. \& Bruning, J. B. Review of the structural and dynamic mechanisms of PPARgamma partial agonism. PPAR Res. 2015, 816856 (2015).

7. Yoshida, H., Matsui, T., Yamamoto, A., Okada, T. \& Mori, K. XBP1 mRNA is induced by ATF6 and spliced by IRE1 in response to ER stress to produce a highly active transcription factor. Cell 107, 881-891 (2001).

8. Cho, Y. M., Kim, D. H., Kwak, S. N., Jeong, S. W. \& Kwon, O. J. X-box binding protein 1 enhances adipogenic differentiation of 3T3-L1 cells through the downregulation of Wnt10b expression. FEBS Lett. 587, 1644-1649 (2013).

9. Cho, Y. M. et al. X-box binding protein 1 is a novel key regulator of peroxisome proliferator-activated receptor gamma2. FEBS J. 281, 5132-5146 (2014).

10. Cho, Y. M. et al. miR-148a is a downstream effector of X-box-binding protein 1 that silences Wnt10b during adipogenesis of 3T3-L1 cells. Exp. Mol. Med. 48, e226 (2016).

11. Park, S. W. et al. The regulatory subunits of PI3K, p85alpha and p85beta, interact with XBP-1 and increase its nuclear translocation. Nat. Med. 16, 429-437 (2010).

12. Lee, J. et al. p38 MAPK-mediated regulation of Xbp1s is crucial for glucose homeostasis. Nat. Med. 17, 1251-1260 (2011).

13. Deng, Y. et al. The Xbp1s/GalE axis links ER stress to postprandial hepatic metabolism. J. Clin. Invest. 123, 455-468 (2013).

14. Gregor, M. F. et al. The role of adipocyte XBP1 in metabolic regulation during lactation. Cell Rep. 3, 1430-1439 (2013).

15. Sha, H. et al. Adipocyte spliced form of X-box-binding protein 1 promotes adiponectin multimerization and systemic glucose homeostasis. Diabetes $\mathbf{6 3}$, 867-879 (2014).

16. Cnop, M., Toivonen, S., Igoillo-Esteve, M. \& Salpea, P. Endoplasmic reticulum stress and elF2alpha phosphorylation: The Achilles heel of pancreatic beta cells. Mol. Metab. 6, 1024-1039 (2017).

17. Bai, L. et al. Dissecting multiple steps of GLUT4 trafficking and identifying the sites of insulin action. Cell. Metab. 5, 47-57 (2007).

18. Ng, Y., Ramm, G., Lopez, J. A. \& James, D. E. Rapid activation of Akt2 is sufficient to stimulate GLUT4 translocation in 3T3-L1 adipocytes. Cell Metab. 7, 348-356 (2008).

19. Tontonoz, P. \& Spiegelman, B. M. Fat and beyond: the diverse biology of PPARgamma. Annu. Rev. Biochem. 77, 289-312 (2008).

20. Maeda, N. et al. PPARgamma ligands increase expression and plasma concentrations of adiponectin, an adipose-derived protein. Diabetes $\mathbf{5 0}$, 2094-2099 (2001).

21. Fu, Y., Luo, N., Klein, R. L. \& Garvey, W. T. Adiponectin promotes adipocyte differentiation, insulin sensitivity, and lipid accumulation. J. Lipid Res. 46, 1369-1379 (2005).

22. Jiang, S. et al. Fibroblast growth factor 21 is regulated by the IRE1alpha-XBP1 branch of the unfolded protein response and counteracts endoplasmic reticulum stress-induced hepatic steatosis. J. Biol. Chem. 289, 29751-29765 (2014).

23. Kharitonenkov, A. et al. FGF-21 as a novel metabolic regulator. J. Clin. Invest. 115, 1627-1635 (2005).

24. Dutchak, P. A. et al. Fibroblast growth factor-21 regulates PPARgamma activity and the antidiabetic actions of thiazolidinediones. Cell 148, 556-567 (2012). 
25. BonDurant, L. D. et al. FGF21 regulates metabolism through adiposedependent and -independent mechanisms. Cell Metab. 25, 935-944 e934 (2017).

26. Madhusudhan, T. et al. Defective podocyte insulin signalling through p85XBP1 promotes ATF6-dependent maladaptive ER-stress response in diabetic nephropathy. Nat. Commun. 6, 6496 (2015).

27. Johnson, J. A., Trasino, S. E., Ferrante, A. W. Jr \& Vasselli, J. R. Prolonged decrease of adipocyte size after rosiglitazone treatment in high- and low-fat-fed rats. Obesity (Silver Spring) 15, 2653-2663 (2007).

28. Hunnicutt, J. W., Hardy, R. W., Williford, J. \& McDonald, J. M. Saturated fatty acidinduced insulin resistance in rat adipocytes. Diabetes 43, 540-545 (1994).

29. Nguyen, M. T. et al. JNK and tumor necrosis factor-alpha mediate free fatty acid-induced insulin resistance in 3T3-L1 adipocytes. J. Biol. Chem. 280 35361-35371 (2005).

30. Hu, M. C. et al. XBP-1, a key $r$ egulator of unfolded protein response, activates transcription of IGF1 and Akt phosphorylation in zebrafish embryonic cell line. Biochem. Biophys. Res. Commun. 359, 778-783 (2007).

31. Takatani, T. et al. IRS1 deficiency protects beta-cells against ER stress-induced apoptosis by modulating SXBP-1 stability and protein translation. Sci. Rep. $\mathbf{6}$ 28177 (2016)

32. Wang, S., Dougherty, E. J. \& Danner, R. L. PPARgamma signaling and emerging opportunities for improved therapeutics. Pharmacol. Res. 111, 76-85 (2016).

33. Muise, E. S. et al. Adipose fibroblast growth factor 21 is up-regulated by peroxisome proliferator-activated receptor gamma and altered metabolic states. Mol. Pharmacol. 74, 403-412 (2008).

34. Choi, J. H. et al. Anti-diabetic drugs inhibit obesity-linked phosphorylation of PPARgamma by Cdk5. Nature 466, 451-456 (2010).
35. Viljanen, A. P. et al. Rosiglitazone treatment increases subcutaneous adipose tissue glucose uptake in parallel with perfusion in patients with type 2 diabetes: a double-blind, randomized study with metformin. J. Clin. Endocrinol. Metab. 90, 6523-6528 (2005).

36. Quinn, C. E., Hamilton, P. K., Lockhart, C. J. \& McVeigh, G. E. Thiazolidinediones: effects on insulin resistance and the cardiovascular system. Br. J. Pharmacol. 153, 636-645 (2008).

37. Arner, P. et al. FGF21 attenuates lipolysis in human adipocytes-a possible link to improved insulin sensitivity. FEBS Lett. 582, 1725-1730 (2008).

38. Nielsen, T. S., Jessen, N., Jorgensen, J. O., Moller, N. \& Lund, S. Dissecting adipose tissue lipolysis: molecular regulation and implications for metabolic disease. J. Mol. Endocrinol. 52, R199-R222 (2014).

39. Hevener, A. L. et al. Macrophage PPAR gamma is required for normal skeletal muscle and hepatic insulin sensitivity and full antidiabetic effects of thiazolidinediones. J. Clin. Invest. 117, 1658-1669 (2007).

40. Odegaard, J. I. et al. Macrophage-specific PPARgamma controls alternative activation and improves insulin resistance. Nature $\mathbf{4 4 7}$ 1116-1120 (2007).

41. Hasegawa-Moriyama, M. et al. Peroxisome proliferator-activated receptorgamma agonist rosiglitazone attenuates postincisional pain by regulating macrophage polarization. Biochem. Biophys. Res. Commun. 426, 76-82 (2012).

42. Wang, N., Liang, H. \& Zen, K. Molecular mechanisms that influence the macrophage m1-m2 polarization balance. Front. Immunol. 5, 614 (2014).

43. Solinas, G. \& Becattini, B. JNK at the crossroad of obesity, insulin resistance, and cell stress response. Mol. Metab. 6, 174-184 (2017). 